\title{
AIDS-Related Non-Hodgkin Lymphoma: Imaging Feature Analysis of 27 Cases and Correlation with Pathologic Findings
}

\author{
Jun Yang', Peng Wang ${ }^{2}$, Zhi-Bin $\mathrm{Lv}^{3}$, Lian-Gui Wei ${ }^{3}$, Yun-Liang $\mathrm{Xu}^{3}$, An Zhou ${ }^{3}$, \\ Dong-Hai $\mathrm{Xu}^{3}$, Da-Qing Ma ${ }^{1 *}$
}

\begin{abstract}
Background: Some tumor types are related to HIV, including non-Hodgkin lymphoma (NHL). The morbidity and mortality of NHL has remained high, even after highly active antiretroviral therapy (HAART) was introduced. We collected cases of AIDS with NHL, and evaluated the imaging features and strategies for diagnosis. Materials and Methods: There were 27 patients with AIDS and tumors confirmed by pathology. There were 9 patients with Burkitt lymphoma, 16 with diffuse large B cell lymphomas (DLBCLs), and 2 with primary central nervous system (PCNS) lymphomas. All of the patients underwent a series of imaging studies. Three radiologists analyzed the images, and any disagreement was discussed until consensus was reached. Results: The radiologic manifestations of AIDS with NHL were mainly masses and lymphadenopathy, 3 patients having one mass and 12 two or more masses. 7 patients had lymphadenopathy in one site and 3patients had lymphadenopathy in two or more sites. Coarse mucosal folds, thickening of the gastrointestinal wall, and lumen narrowing were typical manifestations of NHL within the gastrointestinal tract. There were 4 patients with masses and 5 with lymphadenopathy inthe 9 with Burkitt lymphoma, and 11 patients with masses 5 with lymphadenopathy in the 16 with DLBCLs. Conclusion: NHL is a malignancy that usually occurs in patients with AIDS. Imaging is an important method by which to evaluate lesions, masses, and lymphadenopathy. Fine needle aspiration biopsy and stereotaxis biopsy are useful methods by which to diagnose NHL.
\end{abstract}

Keywords: AIDS - non-Hodgkin lymphoma - radiography - pathology - fine needle aspiration biopsy

Asian Pac J Cancer Prev, 15 (18), 7769-7773

\section{Introduction}

The main clinical manifestation of acquired immunodeficiency syndrome (AIDS) is a negative impact on the immune system. Since the beginning of the HIV epidemic in the 1980s, cancer has been prominent in the spectrum of immunodeficiency-related manifestations. In particular, the risk of Kaposi sarcoma (KS) and nonHodgkin lymphoma (NHL) is considerably higher among HIV-infected persons than healthy persons. The cervical cancer risk is also elevated among HIV-infected women, although to a lesser degree. The availability of highly active antiretroviral therapy (HAART) in 1996 has led to improvements in immune function, but without a corresponding decline in AIDS-related morbidity and mortality (Polesel et al.,2 008; Chun et al., 2010; Beaudrap et al., 2013, Mendes Luz et al., 2014).

\section{Materials and Methods}

Twenty-seven patients with AIDS and NHL were collected in this series between October 2008 and June 2013 in our hospital, and included 22 males and 5 females from 24-72 years of age (mean 843.4 years). All of the patients were positive for HIV antibodies; the diagnosis of AIDS was consistent with the standards of the CDC in the US. There were 27 patients with AIDS and tumors confirmed by pathology, 12 of whom were diagnosed by fine needle aspiration biopsy. There were 9 patients with Burkitt lymphoma and 16 patients with diffuse large $B$ cell lymphoma (DLBCL), affecting the gastrointestinal tract and thorax, including 3 patients with gastric lymphomas and 1 patient with jejunal lymphoma. Two patients had thoracic involvement, including 1 patient with primary effusion lymphoma (PEL). The other 2 patients had CNS lymphomas. The diagnoses were proved by pathologic examination, the lymphomas of the gastrointestinal tract and tumors of the stomach and esophagus were confirmed by gastroscopy, and lymphomas in the thorax were verified by bronchoscopy. Primary effusion lymphoma was proved by biopsy.

Several symptoms were noted in this series of patients. Tumors affecting the gastrointestinal tract, including lymphomas and tumors, were associated with abdominal pain and diarrhea, some patients had dark stools, and weight loss occurred. The patients with a primary effusion

${ }^{I}$ Department of Radiology, Beijing Friendship Hospital, ${ }^{2}$ Department of Pathology, ${ }^{3}$ Department of Radiology, Beijing Ditan Hospital, Institute of Infectious Diseases, Capital Medical University, Beijing, China*For correspondence: madaqing@263.net 
Table 1. Clinical data

\begin{tabular}{|c|c|c|c|c|c|}
\hline Gender & Age & Diagnosis & CD4 cell count & Clinical stage & $\begin{array}{l}\text { Anti-virus therapy } \\
\text { (schedule and time) }\end{array}$ \\
\hline female & 56 & PEL (diffuse large B cell) & 53 cells/ul & AIDS & None \\
\hline female & 40 & gastric NHL (diffuse large B cell) & 38 cells/ul & AIDS & None \\
\hline male & 53 & gastric NHL (diffuse large B cell) & 53 cells/ul & AIDS & None \\
\hline male & 48 & intestinal NHL (diffuse large B B cell) & 178 cells/ul & AIDS & None \\
\hline male & 57 & mediastinal NHL (diffuse large B cell) & 196 cells/ul & AIDS & None \\
\hline male & 72 & gastric NHL (diffuse large B cell) & 250 cells $/ \mathrm{ul}$ & AIDS & None \\
\hline male & 48 & NHL (diffuse large B cell) & 492 cells /ul & AIDS & None \\
\hline female & 40 & NHL (diffuse large B cell) & 113 cells /ul & AIDS & yes \\
\hline male & 55 & NHL (diffuse large B cell) & 102 cells /ul & AIDS & yes \\
\hline male & 37 & NHL (diffuse large B cell) & 72 cells /ul & AIDS & yes \\
\hline male & 51 & NHL (diffuse large B cell) & 550 cells $/ \mathrm{ul}$ & AIDS & None \\
\hline male & 53 & NHL (diffuse large B cell) & 68 cells /ul & AIDS & yes \\
\hline male & 39 & NHL (diffuse large B cell) & 200 cells /ul & AIDS & yes \\
\hline male & 40 & NHL (diffuse large B cell) & 30 cells $/ \mathrm{ul}$ & AIDS & None \\
\hline male & 28 & NHL (diffuse large B cell) & 12 cells /ul & AIDS & None \\
\hline male & 52 & NHL (diffuse large B cell) & 200 cells /ul & AIDS & None \\
\hline male & 40 & Burkitt & 415 cells $/ \mathrm{ul}$ & AIDS & None \\
\hline male & 30 & Burkitt & 136 cells /ul & AIDS & yes \\
\hline male & 36 & Burkitt & 174 cells /ul & AIDS & None \\
\hline male & 47 & Burkitt & 112 cells /ul & AIDS & None \\
\hline male & 41 & Burkitt & 55 cells /ul & AIDS & None \\
\hline male & 48 & Burkitt & 12 cells /ul & AIDS & None \\
\hline male & 33 & Burkitt & 11 cells /ul & AIDS & None \\
\hline male & 32 & Burkitt & 261 cells /ul & AIDS & None \\
\hline female & 40 & Burkitt & 17 cells /ul & AIDS & None \\
\hline male & 24 & PCNS lymphoma & 2 cells $/ \mathrm{ul}$ & AIDS & None \\
\hline female & 32 & PCNS lymphoma & 97 cells /ul & AIDS & None \\
\hline
\end{tabular}

The normal CD4 cell count is 706-1125 cells /ul in our hospital

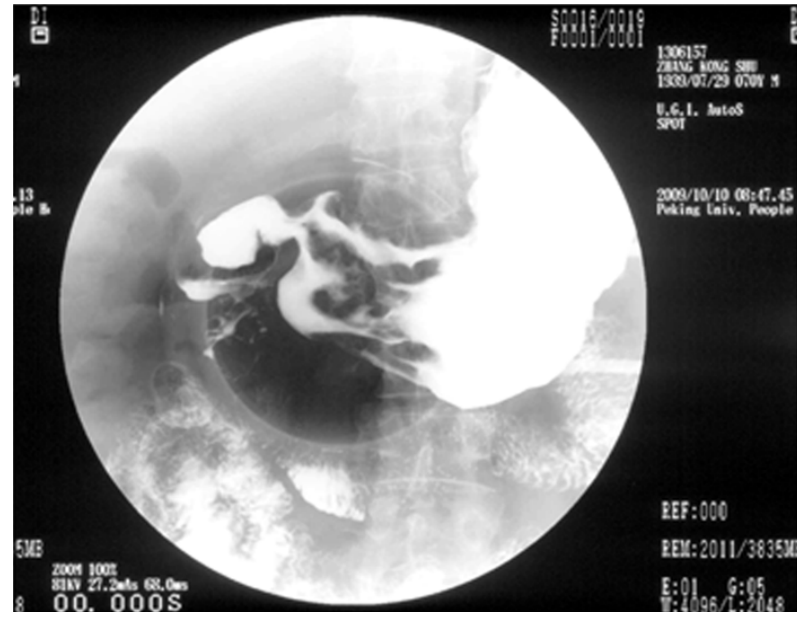

Figure 1. Posteroanterior Radiograph from an upper Gastrointestinal Series in a Patient with Gastric Lymphoma show some of the Mucosal Folds of Gastric Antrum are Evidently Coarse, and irregular, manifest as a linear filling defect, while the local stomach wall is still and cannot expand, and peristalsis disappears.

lymphoma initially had effusions in the thorax and abdominal cavities. The patient's clinical data are shown in Table 1.

All patients had a series of images obtained that were analyzed by three radiologists, including plain films and CT scans of different body sites. Three patients had an upper gastrointestinal series examination; disagreement among the 3 physicians was discussed and a consensus was reached.

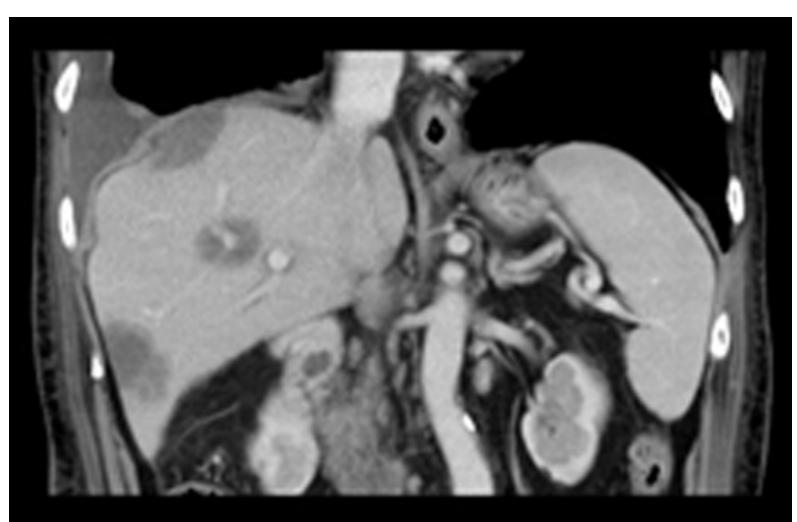

Figure 2. Multiple Focal Liver Lesions in Patients with AIDS-Related NHL appear on Contrast-Enhanced CT Scans as Low-Density Lesions

\section{Results}

The imaging features of AIDS-related non-Hodgkin's lymphoma

In this group of patients, there were 6 cases of NHL affecting the gastrointestinal tract and mediastinum, including gastric lymphomas in 3 patients and jejunal lymphoma in 1 patient; 3 patients had an upper gastrointestinal series examination. The imaging manifestations of these patients were coarse mucosal folds, a narrow lumen, and thickening of the gastrointestinal wall, especially in the patient with a jejunal lymphoma. Two patients with lymphomas had liver infiltration, with multiple local round lesions of lower density, regardless of the plain scan or after enhancement. One patient with 


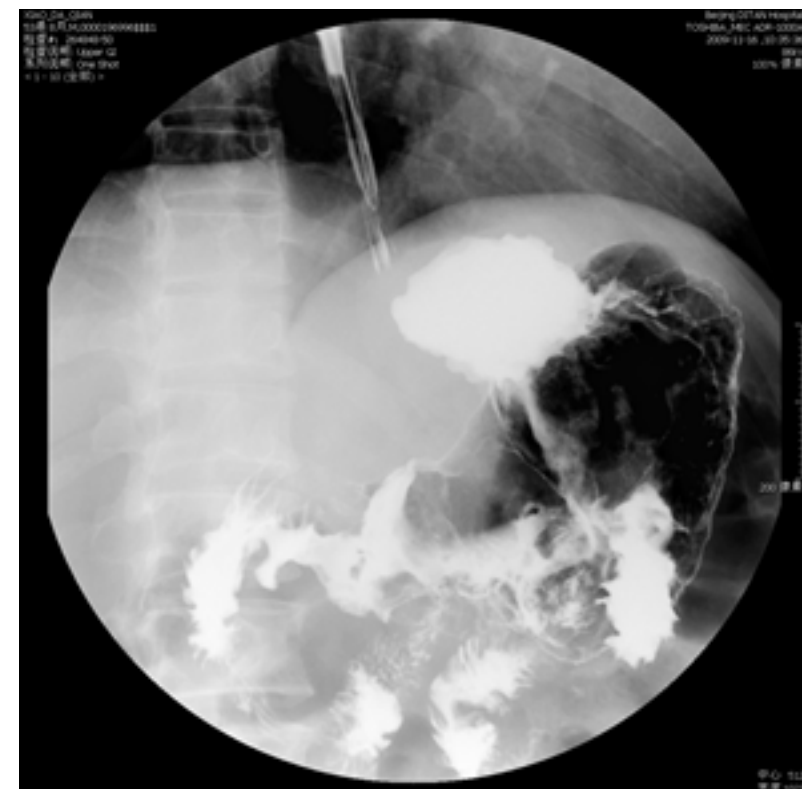

Figure 3. Posteroanterior Radiograph from an Upper Gastrointestinal Series in a Patient with Gastric Lymphoma show the Lumen of the Gastric Body and Antrum is Narrow. The local stomach wall is still and cannot expand, peristalsis disappears, and multiple crater and filling defect is seen, and the duodenal cap is deformationCT shows gastric antrum thickening $(>1.5 \mathrm{~cm})$

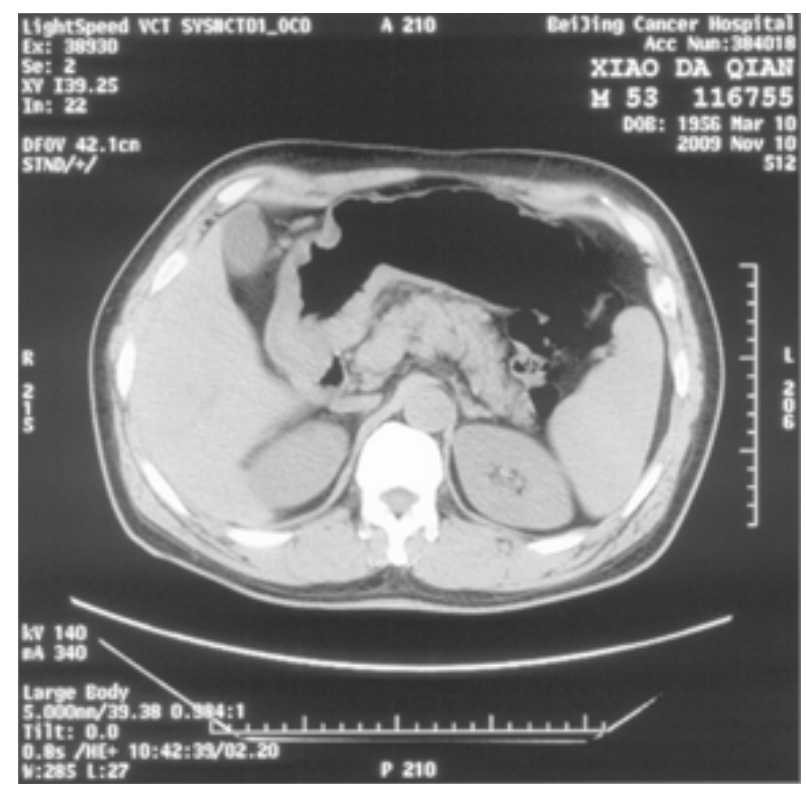

Figure 4. CT Shows Gastric Antrum Thickening (> $1.5 \mathrm{~cm})$

a lymphoma in the thorax had enlarged lymph nodes in the mediastinum. Another patient with a primary effusion lymphoma initially had effusions in the thorax and abdominal cavities, then multiple systems were affected.

The pathology of AIDS-related lymphoma.

\section{Discussion}

Since the beginning of the HIV epidemic in the 1980 , cancer has been prominent in the spectrum of immunodeficiency-related clinical manifestations (Munn et al., 2002; Carbone and Gloghini, 2005; Corti et al., 2005). In particular, the risk of KS and NHL is considerably higher among HIV-infected patients than healthy patients. The risk of cervical cancer also is also increased among HIV-infected women, although to a lesser degree than KS and NHL.

Recently, Ramanathan et al. reported an analysis of oral extronodal NHL cases (Ramanathan et al., 2014). In their paper, the authors selected forty two patients diagnosed with extranodal oral cavity NHL. The authors performed a thorough analysis of these cases and found that these lesions generally appear as a painless swelling and they mostly occur in men. They also identified these cases as either B-cell or T-cell NHL and investigated their occurrence in different ethnic groups. Although these results will certainly help understand this serious disease and increase the awareness and education of NHL, discussion of new diagnosis techniques and the correlation between NHL and other serious diseases such as AIDS will further help healthcare professionals to treat these patients. We believe our paper will serve this purpose.

NHL, KS, and cervical cancers are considered to be AIDS-defining conditions, and referred to as AIDSdefining malignancies. All three cancers are caused by oncogenic viruses: specifically, human herpesvirus 8 causes KS; and Epstein Barr virus (EBV) causes the two most common AIDS-associated NHL subtypes (DLBCL and central nervous system NHL). A major feature of these cancers in HIV-infected patients is the association with immunosuppression. For KS and EBV-related NHL subtypes, the risk increases as the CD4 count decreases.

In the HAART era, infection-related carcinoma would likely become an increasingly important complication of longevity amongst HIV infection patients. NHL is the second most common tumor in people with AIDS; highgrade tumors are designated as AIDS-defining, including small non-cleaved lymphoma (Burkitt or non-Burkitt), large cell lymphoma (Milling et al., 2005), immunoblastic lymphoma (Brimo et al., 2007), and PCNS lymphoma. NHL previously accounted for approximately $3 \%$ of AIDS-defining clinical conditions, but this proportion had declined. The incidence of PCNS lymphoma was highest; other types of lymphomas appear as case reports in the literature (Rich et al., 1992; Cappell and Botros, 1994; Rizzi et al., 2001; Doweiko et al., 2004; Khan et al., 2006; Mohan et al., 2007; Mani et al., 2008; Rezende et al., 2009; Kalogeropoulos et al., 2009; Li and Cheng, 2011). Previously, NHL accounted for approximately $3 \%$ of AIDS-defining clinical conditions, but this percentage has declined. The risk of developing NHL is approximately six-fold higher than among healthy people. The risk factors for AIDS-associated NHL increase with age and are higher in men than women. EBV was detected in approximately $50 \%$ of all AIDS-associated NHL, while including nearly $100 \%$ of PCNS lymphomas (Marcelo et al., 2006). The characteristics of lymphomas in HIVseropositive patients included high-grade histology, rapid spread, widespread involvement, poor response to chemotherapy, and very short survival. The introduction of HAART radically changed the clinical spectrum of HIV and AIDS-associated tumors. The prognosis of AIDS-associated NHL has improved in the era of 
HAART, in part due to higher mean CD4 counts at the time of NHL diagnosis, which has allowed patients to better tolerate chemotherapy. As a special type of NHL, primary effusion lymphoma is a rare lymphoma with a tropism for serous body cavities (pleural, peritoneal, or pericardial cavities), although dissemination to or initial manifestation in viscera are not clear. Primary effusion lymphomas typically express a high HHV-8 copy number, and frequently are co-infected with EBV; the proportion of NHL due to primary effusion lymphomas is not known. HIV-associated NHL may present as a systemic disease in $80 \%$ of patients, but $20 \%$ are localized to CNS or body cavities (Senocak et al., 2010; Latta et al., 2010). Moreover, $80 \%$ also involve extranodal sites, such as the bone marrow, CNS, gastrointestinal tract, and liver. Most lymphomas in this population had an aggressive histopathology and are of the B-cell type.

The lung and mediastinum are major targets for HIV infection and the onset of AIDS is often associated with severe thoracic complications. Primary mediastinal large B-cell lymphomas typically occur in young women in the third-to-fifth decades of life, originating from the anterior and superior mediastinum, and are locally aggressive and usually did not involve bone narrow or extrathoracic structures; patients usually present with symptoms from locally advanced disease. Patients infected with HIV who also develop primary gastric lymphomas usually present with abdominal pain, tenderness, weight loss, and bleeding. This lymphoma tends to have a worse prognosis with a reported survival of only 6-9 months. The prognosis of these lymphomas may be impacted adversely by other prognostic factors, including advanced HIV infection or low CD4 count. Most HIV-related gastric lymphomas are of a diffuse large B-cell type, as the patients in this group. These lymphomas are immunophenotypically similar to lymphomas of comparable morphology occurring in the non-HIV-infected population. An important feature of gastrointestinal lymphomas is that the gastrointestinal tract usually provides the most straightforward and simple method of diagnosis. Barium radiography and CT might be helpful in the evaluation of gastrointestinal lymphomas, and endoscopy with biopsy is usually diagnostic. There are 3 main morphologic patterns in the hepatic NHL (large solitary masses $(>4 \mathrm{~cm})$, multiple focal nodules, and diffuse infiltrating disease. The first two patterns are the most common. Single or multiple focal liver lesions in patients with AIDS-related NHL appear on both unenhanced and contrast-enhanced CT scans as low-density lesions. In this series of patients, the characters of NHL is as follows: all cases were B cells; all patients had not taken HAART; the CD4 count was very low; all patients had AIDS ( 5 patients had extranodal involvement andone-half of the patients had multiple system involvement.

In summary, imaging is valuable for diagnosis and differential diagnosis of AIDS with tumors (Davison et al., 2008; Carbone et al., 2010; Evans et al., 2011; Jessica et al., 2011; Liu, 2012), and facilitates staging and observing a therapeutic effect; however, biopsy is needed to confirm the diagnosis. NHL is a malignant carcinoma that usually occurs in patients with AIDS, and imaging is a good method to manifest lesions, especially in the early stage. Diagnosis in the early stage is important for treating effect and improving the prognosis in the HARRT era. Fine needle aspiration biopsy is a useful method of diagnosis.

\section{Acknowledgements}

This study don't have any grant support.

\section{References}

Beaudrap PD, Boulle C, Lewden C, et al (2013) Morbidity After Antiretroviral Therapy Initiation in HIV-1-Infected Children in West Africa: Temporal Trends and Relation to CD4 Count. Ped Infect Dis J, 32, 354-60.

Busi Rizzi E, Schinina V, Cristofaro M, et al (2001). NonHodgkin's lymphoma of the liver in patients with AIDS: sonographic, CT, and MRI findings. J Clin Ultrasound, 29, 125-9.

Brimo F, Michel RP, Khetani K, et al (2007). Primary effusion lymphoma: a series of 4 cases and review of the literature with emphasis on cytomorphologic and immunocytochemical differential diagnosis. Cancer, 111, 224-33.

Carbone A, Cesarman E, Gloghini A, et al (2010). Understanding pathogenetic aspects and clinical presentation of primary effusion lymphoma through its derived cell lines. AIDS, 24, 479-90.

Cappell MS, Botros N (1994). Predominantly gastrointestinal symptoms and signs in 11 consecutive AIDS patients with gastrointestinal lymphoma: a multicenter, multiyear study including $763 \mathrm{HIV}$-seropositive patients. Am J Gastroenterol, 89, 545-9.

Chun C, Lanfang X, Donald A, et al (2010). Survival of nonHodgkin lymphoma patients with and without HIV infection in the era of combined antiretroviral therapy. AIDS, 24, $1765-70$.

Carbone A, Gloghini A (2005). AIDS-related lymphomas: from pathogenesis to pathology. British Journal of Haematology, 130, 662-70.

Corti M, Villafane MF, Trione N, et al (2005). Primary pulmonary AIDS-related lymphoma. Rev Inst Med Trop, 47, 231-4.

Davison JM, Subramaniam RM, Surasi DS, et al (2011). FDG PET/CT in patients with HIV. AJR, 197, 284-94.

Doweiko J, Dezube BJ, Pantanowitz L (2004). Unusual sites of Hodgkin's lymphoma: CASE 1. HIV-associated Hodgkin's lymphoma of the stomach. J Clin Oncol, 22, 4227-8.

Evans WC, Gilmore D, English J (2011). The role of PET and PET-CT in managing the care of lymphoma patients. $\mathrm{J} \mathrm{Nucl}$ Med Technol, 39,190-4.

Khan F, Bauer F, Gazi G, et al (2006). Regression of large B-cell non-Hodgkin's lymphoma of stomach with HAART: case report and review. Leuk Lymphoma, 47, 750-4.

Kalogeropoulos IV, Chalazonitis AN, Tsolaki S, et al (2009). A case of primary isolated non-Hodgkin's lymphoma of the esophagus in an immunocompetent patient. World $J$ Gastroenterol, 15, 1901-3.

Latta S, Myint ZW, Jallad B (2010). Primary central nervous system T-cell lymphoma in AIDS patients case report and literature review. Current Oncology, 17, 63-6.

Liu Y (2012). Concurrent FDG avid nasopharyngeal lesion and generalized lymphadenopathy on PET-CT imaging is indicative of lymphoma in patients with HIV infection. AIDS Research Treatment.???

Li HJ, Cheng JL (2011). AIDS complicated with intestinal lymphoma X-ray radiology, CT scan and pathological findings. Chinese Medical Journal, 124, 1427-30. 
Mani D, Guinee D, Aboulafia D (2008). AIDS-associated plasmablastic lymphoma presenting as a poorly differentiated esophageal tumor: a diagnostic dilemma. World J Gastroenterol, 14, 4395-9.

Marcelo C, Maria F. Villafane F, et al (2006). Central nervous system involvement in Hodgkin's lymphoma associated with Epstein-Barr virus. Braz J Infect Dis, 10, 403-5.

Mendes Luz P, Bruyand M, Ribeiro S, et al (2014). AIDS and non-AIDS severe morbidity associated with hospitalizations among HIV-infected patients in two regions with universal access to care and antiretroviral therapy, France and Brazil, 2000-2008: hospital-based cohort studies. BMC Infect Dis, 14, 278-82.

Munn S (2002). Imaging HIV/AIDS. Burkitt's lymphoma. AIDS Patient Care STDS, 16, 395-9.

Milling DL, Lazarchick J, Chaudhary UB (2005). Primary mediastinal large B-cell lymphoma in an HIV-infected patient. Am J Med Sci, 329, 136-8.

Mohan H, Bal A, Garg S, et al (2007). Cytomegalovirusassociated pseudotumor simulating gastric malignancy in acquired immunodeficiency syndrome: a case report with review of literature. Jpn J Infect Dis, 60, 134-6.

Polesel J, Clifford GM, Rickenbach M, et al (2008). NonHodgkin lymphoma incidence in the Swiss HIV Cohort Study before and after highly active antiretroviral therapy. AIDS, 22, 301-6.

Ramanathan A, Mahmoud HRR, Hui LP, et al (2014). Oral extranodal non Hodgkin's lymphoma: series of forty two cases in Malaysia, Asian Pac J Cancer Prev, 15, 1633-37.

Rich JD, Crawford JM, Kazanjian SN, et al (1992). Discrete gastrointestinal mass lesions caused by cytomegalovirus in patients with AIDS: report of three cases and review. Clin Infect Dis, 15, 609-14.

Rezende REF, Mantelmacher M, Ferreira SDC, et al (2009). Clinical, endoscopic and prognostic aspects of primary gastric non-Hodgkin's lymphoma associated with acquired immunodeficiency syndrome. Braz J Infect Dis, 13, 2-4.

Senocak E, Oguz KK, Ozgen B, et al (2010). Imaging features of CNS involvement in AIDS. Diagn Interv Radiol, 16, 193-200.

Zacharia TT, Law M, Naidich TP, et al (2008). Central nervous system lymphoma characterization by diffusion-weighted imaging and MR spectroscopy. J Neuroimaging, 18, 411-7. 